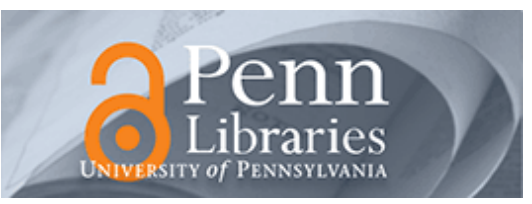

University of Pennsylvania ScholarlyCommons

6-2-2009

\title{
Mechanical stiffness and dissipation in ultrananocrystalline diamond microresonators
}

Vivekananda P. Adiga

University of Pennsylvania, adiga@seas.upenn.edu

A V. Sumant

Argonne National Laboratory

S Suresh

Innovative Micro Technolog

C Gudeman

Innovative Micro Technolog

O Auciello

Argonne National Laboratory

Follow this and additional works at: https://repository.upenn.edu/mse_papers

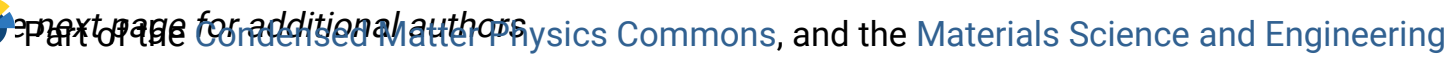
Commons

\section{Recommended Citation}

Adiga, V. P., Sumant, A. V., Suresh, S., Gudeman, C., Auciello, O., Carlisle, J. A., \& Carpick, R. W. (2009). Mechanical stiffness and dissipation in ultrananocrystalline diamond microresonators. Retrieved from https://repository.upenn.edu/mse_papers/174

Copyright 2009 American Physical Society. Reprinted from:

Mechanical stiffness and dissipation in ultrananocrystalline diamond microresonators. V. P. Adiga, A. V. Sumant, S.

Suresh, C. Gudeman3, O. Auciello, J. A. Carlisle, and R. W. Carpick. Phys. Rev. B 79, 245403 (2009)

Publisher URL: http://link.aps.org/doi/10.1103/PhysRevB.79.245403

DOI: 10.1103/PhysRevB.79.245403

This paper is posted at ScholarlyCommons. https://repository.upenn.edu/mse_papers/174

For more information, please contact repository@pobox.upenn.edu. 


\title{
Mechanical stiffness and dissipation in ultrananocrystalline diamond microresonators
}

\begin{abstract}
We have characterized mechanical properties of ultrananocrystalline diamond UNCD thin films grown using the hot filament chemical vapor deposition HFCVD technique at $680^{\circ} \mathrm{C}$, significantly lower than the conventional growth temperature of $800^{\circ} \mathrm{C}$. The films have $4.3 \% \mathrm{sp} 2$ content in the near-surface region as revealed by near edge $x$-ray absorption fine structure spectroscopy. The films, $1 \mathrm{~m}$ thick, exhibit a net residual compressive stress of $3701 \mathrm{MPa}$ averaged over the entire $150 \mathrm{~mm}$ wafer. UNCD microcantilever resonator structures and overhanging ledges were fabricated using lithography, dry etching, and wet release techniques. Overhanging ledges of the films released from the substrate exhibited periodic undulations due to stress relaxation. This was used to determine a biaxial modulus of $8382 \mathrm{GPa}$. Resonant excitation and ring-down measurements in the $\mathrm{kHz}$ frequency range of the microcantilevers were conducted under ultrahigh vacuum UHV conditions in a customized UHV atomic force microscope system to determine Young's modulus as well as mechanical dissipation of cantilever structures at room temperature. Young's modulus is found to be $79030 \mathrm{GPa}$. Based on these measurements, Poisson's ratio is estimated to be 0.0570 .038 . The quality factors $Q$ of these resonators ranged from 5000 to 16000 . These $Q$ values are lower than theoretically expected from the intrinsic properties of diamond. The results indicate that surface and bulk defects are the main contributors to the observed dissipation in UNCD resonators.
\end{abstract}

\section{Keywords}

resonators, ultrananocrystalline diamond, dissipation, modulus, poisson's ratio

\section{Disciplines}

Condensed Matter Physics | Materials Science and Engineering

\section{Comments}

Copyright 2009 American Physical Society. Reprinted from:

Mechanical stiffness and dissipation in ultrananocrystalline diamond microresonators. V. P. Adiga, A. V. Sumant, S. Suresh, C. Gudeman3, O. Auciello, J. A. Carlisle, and R. W. Carpick. Phys. Rev. B 79, 245403 (2009)

Publisher URL: http://link.aps.org/doi/10.1103/PhysRevB.79.245403

DOI: 10.1103/PhysRevB.79.245403

\section{Author(s)}

Vivekananda P. Adiga, A V. Sumant, S Suresh, C Gudeman, O Auciello, J A. Carlisle, and Robert W. Carpick 


\title{
Mechanical stiffness and dissipation in ultrananocrystalline diamond microresonators
}

\author{
V. P. Adiga, ${ }^{1}$ A. V. Sumant, ${ }^{2}$ S. Suresh, ${ }^{3}$ C. Gudeman, ${ }^{3}$ O. Auciello, ${ }^{2,4}$ J. A. Carlisle, ${ }^{5}$ and R. W. Carpick ${ }^{1,6}$ \\ ${ }^{1}$ Department of Materials Science and Engineering, University of Pennsylvania, Philadelphia, Pennsylvania 19104, USA \\ ${ }^{2}$ Center for Nanoscale Materials, Argonne National Laboratory, Argonne, Illinois 60439, USA \\ ${ }^{3}$ Innovative Micro Technology, Santa Barbara, California 93117, USA \\ ${ }^{4}$ Materials Science Division, Argonne National Laboratory, Argonne, Illinois 60439, USA \\ ${ }^{5}$ Advanced Diamond Technologies, Romeoville, Illinois 60446, USA \\ ${ }^{6}$ Department of Mechanical Engineering and Applied Mechanics, University of Pennsylvania, Philadelphia, Pennsylvania 19104, USA \\ (Received 27 January 2009; published 2 June 2009)
}

\begin{abstract}
We have characterized mechanical properties of ultrananocrystalline diamond (UNCD) thin films grown using the hot filament chemical vapor deposition (HFCVD) technique at $680{ }^{\circ} \mathrm{C}$, significantly lower than the conventional growth temperature of $\sim 800{ }^{\circ} \mathrm{C}$. The films have $\sim 4.3 \% s p^{2}$ content in the near-surface region as revealed by near edge $\mathrm{x}$-ray absorption fine structure spectroscopy. The films, $\sim 1 \mu \mathrm{m}$ thick, exhibit a net residual compressive stress of $370 \pm 1 \mathrm{MPa}$ averaged over the entire $150 \mathrm{~mm}$ wafer. UNCD microcantilever resonator structures and overhanging ledges were fabricated using lithography, dry etching, and wet release techniques. Overhanging ledges of the films released from the substrate exhibited periodic undulations due to stress relaxation. This was used to determine a biaxial modulus of $838 \pm 2 \mathrm{GPa}$. Resonant excitation and ring-down measurements in the $\mathrm{kHz}$ frequency range of the microcantilevers were conducted under ultrahigh vacuum (UHV) conditions in a customized UHV atomic force microscope system to determine Young's modulus as well as mechanical dissipation of cantilever structures at room temperature. Young's modulus is found to be $790 \pm 30 \mathrm{GPa}$. Based on these measurements, Poisson's ratio is estimated to be $0.057 \pm 0.038$. The quality factors $(Q)$ of these resonators ranged from 5000 to 16000 . These $Q$ values are lower than theoretically expected from the intrinsic properties of diamond. The results indicate that surface and bulk defects are the main contributors to the observed dissipation in UNCD resonators.
\end{abstract}

DOI: $10.1103 /$ PhysRevB.79.245403

\section{INTRODUCTION}

Nanocrystalline materials present a rich set of opportunities to explore the physics governing how defects such as grain boundaries, impurities, and dangling bonds affect the mechanical performance. Diamond, the stiffest material known, can be grown as a conformal thin film on a variety of surfaces in a form known as ultrananocrystalline diamond (UNCD) (grain size $\sim 2-5 \mathrm{~nm}$ ). ${ }^{1-3}$ UNCD films were originally grown using microwave plasma chemical vapor deposition (MPCVD), and Young's modulus ${ }^{4,5}$ and hardness ${ }^{5}$ of films grown using this well-established recipe have been measured. These films exhibit smooth surfaces ${ }^{1}$ and mechanical properties close to single-crystal diamond despite the high volume fraction of grain boundaries and the presence of atoms such as hydrogen. ${ }^{6}$ However, Poisson's ratio of UNCD has never been reported, and measurements of mechanical dissipation (quality factor) are scant. While modest deviations in the elastic properties (modulus, Poisson's ratio) from the values for bulk diamond can be expected due to the polycrystalline nature of UNCD or the possibility of defects, the quality factor is far more difficult to predict since mechanical dissipation has a large number of possible sources which are often very sensitive functions of several material parameters.

In general, UNCD and nanocrystalline diamond (grain size $\sim 10 \mathrm{~nm}-100 \mathrm{~nm}$ ) thin films exhibit a unique combination of properties suitable for applications to microelectromechanical/nanoelectromechanical system (MEMS/NEMS) devices, ${ }^{2}$ such as radio frequency MEMS/ NEMS resonators. ${ }^{7-11}$ This is due to their superior physical properties such as a high Young's modulus, their stable and
PACS number(s): 62.25. $-\mathrm{g}, 62.40 .+\mathrm{i}, 62.30 .+\mathrm{d}, 62.20 . \mathrm{dj}$

inert surface with low adhesion to other materials, and their good tribological performance. ${ }^{12-14}$ High-frequency devices based on MEMS or NEMS structures have a large surface to volume ratio and can have high dissipation if surface effects dominate the loss mechanisms. ${ }^{15,16}$ However, the surface stability and high stiffness of UNCD films suggest it has potential for high-frequency resonators with high quality factors.

The ability to grow uniform and smooth UNCD films over a large area at relatively low temperatures $\left(\leq 500{ }^{\circ} \mathrm{C}\right)$ has enabled the cointegration of complementary metaloxide-semiconductor (CMOS) electronics with UNCD MEMS devices (e.g., resonators and switches). ${ }^{1,2}$ The mechanical properties of UNCD films grown at low temperatures play an important role in the suitability of these films for applications involving monolithic integration of MEMS/ NEMS and CMOS devices. In this paper, we present studies of Young's modulus, Poisson's ratio, and mechanical dissipation of UNCD films grown at $680{ }^{\circ} \mathrm{C}$ using the hot filament chemical vapor deposition (HFCVD) technique. We determined the Young's modulus of these films by fabricating UNCD microcantilevers and measuring the resonance frequency of the fundamental flexural mode of the cantilevers. Underetching of the UNCD films to produce overhanging ledges resulted in periodic wrinkles due to residual compressive stress in the film. The amplitude and wavelength of the fully relaxed overhanging portion were measured to determine the biaxial modulus of the film and, by comparison with the Young's modulus, Poisson's ratio. Ring-down measurements of resonantly excited cantilevers were used to determine mechanical dissipation in the UNCD at $\sim \mathrm{kHz}$ frequencies. 


\section{DISSIPATION IN MICROCANTILEVERS}

Dissipation in resonators can be intrinsic or extrinsic in origin. Viscous damping from a surrounding fluid medium (e.g., air), clamping losses, or dissipation due to the measuring system itself are examples of extrinsic losses. Under UHV conditions, air damping is eliminated. ${ }^{17}$ A lower bound on dissipation from the measuring system can be determined by carrying out a measurement on a high $Q$ structure.

There are several models ${ }^{18-20}$ for quality factors limited by clamping losses, represented as $Q_{\text {clamping, for out of plane }}$ flexural cantilever resonators attached to supports of different dimensions. Jimbo et $a .^{20}$ predicted the quality factor limited by clamping loss of cantilever beam of infinite width attached to semi-infinite base to be given by

$$
Q_{\text {clamping }} \propto\left(\frac{L}{t}\right)^{3},
$$

where $L$ is the length and $t$ is the thickness of the cantilever. Later Cross et al. ${ }^{18}$ proposed equations for out of plane flexural resonators of finite width attached to thickness-matching bases. For the base thickness $\left(t_{b}\right)$ smaller than the wavelength of the elastic wave in the base $\left(\lambda_{b}\right)$, quality factors limited by clamping losses to the thickness-matching base are proportional to the aspect ratio of the levers $(L / w) .{ }^{18,19}$ However this model does not take into account the fact that thickness-matching bases can be overhangs where the energy can be reflected back to the oscillator. ${ }^{18}$ Photiadis et al. ${ }^{19}$ developed a model for clamping losses of flexural cantilever resonators with finite $\left(t_{b}<\lambda_{b}, t_{b} \geq t\right)$ and semi-infinite $\left(t_{b}\right.$ $\left.>\lambda_{b}\right)$ bases. For finite $\left(t_{b}<\lambda_{b}\right)$ base, ${ }^{19}$

$$
Q_{\text {clamping }} \propto \frac{L}{w}\left(\frac{t_{b}}{t}\right)^{2} .
$$

Intrinsic dissipation processes relevant to UNCD cantilevers include thermoelastic dissipation (TED), ${ }^{21-23}$ phononphonon dissipation, ${ }^{24}$ and strain-assisted relaxation of defects. ${ }^{25}$ Thermoelastic dissipation occurs mainly due to the temperature gradients set up by the oscillations in the solid which causes local volume changes. This results in irreversible heat flow across the temperature gradient resulting in dissipation of the mechanical energy. Zener et al. ${ }^{21}$ developed an approximate equation for thermoelastic dissipation, which for flexural beams is given by

$$
Q^{-1}=\frac{E \alpha^{2} T}{\rho C_{P}} \frac{\omega \tau_{z}}{1+\left(\omega \tau_{z}\right)^{2}},
$$

where $\alpha, C_{P}$, and $T$ are the coefficient of thermal expansion, specific heat, and temperature of the material, respectively, $\omega$ is the oscillation frequency, and $\tau_{z}$ is the relaxation time for TED given by

$$
\tau_{z}=\frac{t^{2}}{\pi^{2} D},
$$

where $D$ is the thermal diffusivity. For UNCD, $D$ will be much smaller than single-crystal diamond due to the lower thermal conductivity of $\mathrm{UNCD}^{26}$ caused by the large fraction of grain boundaries in UNCD. Precise solutions for the ther- moelastic dissipation in thin beams were developed by Lifshitz et al. ${ }^{22}$ However, TED calculated from an exact solution derived by Lifshitz and an approximate solution given by Zener are in close agreement. TED in polycrystalline materials is discussed by Srikar et al. ${ }^{23}$ For low-frequency UNCD microcantilevers, TED due to Zener damping will be much larger than TED due to intracrystalline and intercrystalline damping mechanisms which are active at $\mathrm{GHz}$ frequencies. This is due to the extremely high thermal conductivity of individual diamond grains and their nanoscale dimension. $^{23}$

Another important form of intrinsic dissipation occurs due to the interaction between thermal phonons and the mechanical oscillations of the resonator. If the wavelength of the acoustic wave is much larger than the mean free path of the thermal phonons, then this low-frequency mechanical oscillation locally perturbs the thermal phonon distributions away from equilibrium. ${ }^{25}$ Restoring this equilibrium cause dissipation in the oscillating beam. Dissipation by such an interaction is given by

$$
Q_{\mathrm{ph}-\mathrm{ph}}^{-1}=\frac{C T \gamma^{2}}{\rho v^{2}} \frac{\omega \tau_{\mathrm{ph}}}{1+\left(\omega \tau_{\mathrm{ph}}\right)^{2}},
$$

where $\gamma$ is Grüneisen's constant, $v$ is the sound velocity, $C$ is the heat capacity per unit volume, and $\tau_{\mathrm{ph}}$ is the phonon relaxation time, which for flexural beams is given by

$$
\tau_{\mathrm{ph}}=\frac{3 \kappa}{C v_{D}^{2}},
$$

where $v_{D}$ is the Debye sound velocity, which depends of the transverse and longitudinal sound velocities, ${ }^{25}$ and $\kappa$ is the thermal conductivity.

However, dissipation in most oscillators, including cantilevers, is usually dominated by intrinsic dissipation due to the stress-assisted relaxation of defects. When subjected to stress, impurity atoms, dislocations, and other defects at grain boundaries and surfaces undergo a transition from one state to another, similar to two level systems. Dissipation in such systems exhibit Debye peaks with a characteristic relaxation time $\tau$ which is unique to that transition. The frequency-dependent quality factor limited by such transitions is given by ${ }^{27}$

$$
Q_{\text {Defect }}=A\left[\frac{\omega \tau}{1+(\omega \tau)^{2}}\right]^{-1},
$$

where $A$ is a constant which depends on the nature of the defect and defect concentration (number of defects per unit volume) and $\tau$ is the relaxation time which follows the Arrhenius relation given by ${ }^{27}$

$$
\frac{1}{\tau}=\frac{1}{\tau_{0}} \exp \left(-\frac{E_{A}}{k_{B} T}\right),
$$

where $E_{A}$ is the activation energy for the process, $k_{B}$ is the Boltzmann constant, and $\tau_{0}$ is the characteristic atomic vibration frequency.

Another important form of intrinsic dissipation occurs at interfaces of high-frequency (rf) resonators, especially at the surfaces of resonators. Dissipation at surfaces could domi- 


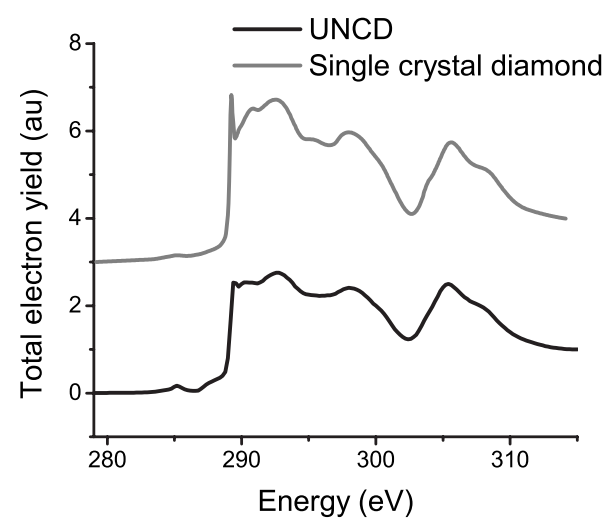

FIG. 1. NEXAFS spectra from a H-terminated UNCD film and single-crystal diamond (offset for clarity). UNCD exhibits a $s p^{2}$ peak at $285 \mathrm{eV}$, a diminished exciton peak at $289 \mathrm{eV}$, and shallower band gap at $302 \mathrm{eV}$, all due to the fractional presence of nondiamond bonding.

nate the losses in oscillators that have a high surface-tovolume ratio ${ }^{15,16}$ and could arise from the presence of a large number of defects at the surface, the presence of extraneous material (contamination), defects induced by processing steps such as etching, or simply roughness on the surfaces of the structure.

\section{FILM GROWTH AND CHARACTERIZATION}

UNCD films (known as Aqua 25 by Advanced Diamond Technologies Inc.) were grown using the HFCVD technique at $680{ }^{\circ} \mathrm{C}$ on silicon wafers (diameter $=150 \mathrm{~mm}$ ) using a predominantly methane/hydrogen growth chemistry. Silicon wafer were ultrasonically seeded with ultradispersive detonation diamond (UDD) powder solution before the growth. The average film thickness was measured by the NANOSPEC technique to be $1.002 \mu \mathrm{m}$, uniform to within $11 \%$ across the wafer with the thickness greatest at the center. These films exhibited a residual compressive stress of $370 \pm 1$ MPa measured using a Tencor Flexus 2320A stress measurement tool. NEXAFS spectroscopy was used to determine the chemical bonding nature of the films, especially for the presence of $s p^{3}$ and $s p^{2}$ content in the near-surface region (top $\sim 4 \mathrm{~nm}$ ) of the films. ${ }^{28}$ A NEXAFS spectrum taken on the topside of a hydrogen-terminated UNCD film grown with the conditions indicated above is shown in Fig. 1 (black curve), where a NEXAFS spectrum from a single-crystal diamond is shown for comparison (gray curve). Characteristic diamond features observed in Fig. 1 include the exciton peak at $289.3 \mathrm{eV}$ and second band gap at $\sim 302 \mathrm{eV}$ characteristic of diamond. A small amount of non-diamond bonding in the UNCD film is revealed by the presence of $s p^{2}$-bonded carbon corresponding to the peak in the electron yield centered at $285.0 \mathrm{eV}$. Based on NEXAFS measurements on HFCVD grown UNCD films and the corresponding reference spectra obtained on single-crystal diamond and highly oriented pyrolytic graphite (HOPG), we estimate the $s p^{2}$ content of the films to be $5.8 \%$ using the normalization method described by Lenardi et al. ${ }^{28}$ The $s p^{2}$ content observed in the HFCVD UNCD films stud-

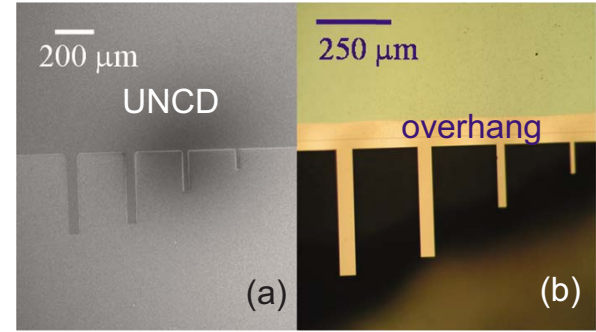

FIG. 2. (Color online) (a) SEM image of unreleased UNCD cantilever die which features four different cantilevers with lengths ranging from 100 to $400 \mu \mathrm{m}$, with a thickness of $1 \mu \mathrm{m}$. (b) Optical microscope image of the back-cleaved cantilever die.

ied here is similar to the $2-5 \% s p^{2}$ content observed in MPCVD films grown at $800{ }^{\circ} \mathrm{C} .{ }^{3}$ Most of this $s p^{2}$-bonded carbon is present at the grain boundaries; some fraction may be due to adsorbates and surface reconstruction. Hydrogen termination $^{29}$ of the surface using a hot filament process resulted in the reduction in $s p^{2}$ content in the near-surface region to $4.3 \%$. Thus, this percentage represents a likely lower bound for the $s p^{2}$ content of the bulk of the film assuming a uniform film structure with thickness. The UNCD films studied here exhibit an RMS roughness of $\sim 10 \mathrm{~nm}$ over $10 \times 10 \mu \mathrm{m}^{2}$ area measured by tapping mode AFM imaging.

\section{FABRICATION AND EXPERIMENTAL SETUP}

For cantilever fabrication, a thick sputtered oxide was deposited on the UNCD surface, and subsequently patterned and etched to serve as a hard mask for etching the UNCD layer to produce the cantilevers. The UNCD layer was etched using oxygen-based plasma in a reactive ion etching (RIE) process to define the UNCD cantilevers. The wafers were diced to commercial AFM chip specifications (4.4 $\times 1.6 \mathrm{~mm}^{2}$ dies) to enable insertion into commercial AFMs for measuring the cantilever properties. Each die contained four cantilevers as shown in the SEM image in Fig. 2(a), with the cantilever lengths of 100, 250, 300, and $400 \mu \mathrm{m}$. The length-to-width ratio is 7.5 for all the cantilevers. The oxide hard mask and sacrificial oxide underneath the cantilevers were subsequently wet etched simultaneously, using a $49 \%$ hydrofluoric acid (HF) by volume solution. The substrates were cleaved so that the cantilevers did not have any substrate material underneath them [Fig. 2(b)]. This was done to ensure that reflected light was sampled only from the cantilever and not from the more reflective Si substrate. Dies

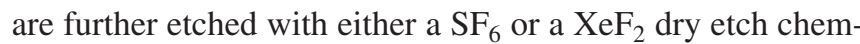
istry to study the effect of overhang on mechanical properties. The thickness of each cantilever was measured by SEM and ranged from $820 \mathrm{~nm}$ to $1.01 \mu \mathrm{m}$. The minor differences in thickness are from thickness variation of the film as deposited or due to subsequent processing. Dies were then cleaned using a piranha solution (3:1 concentrated sulfuric acid to $30 \%$ hydrogen peroxide solution) before being tested under UHV $\left(2 \times 10^{-10}\right.$ Torr $)$ conditions on a custom-built AFM stage inserted into a RHK UHV 350 AFM. The AFM stage consisted of a piezoceramic glued onto an invar holder 

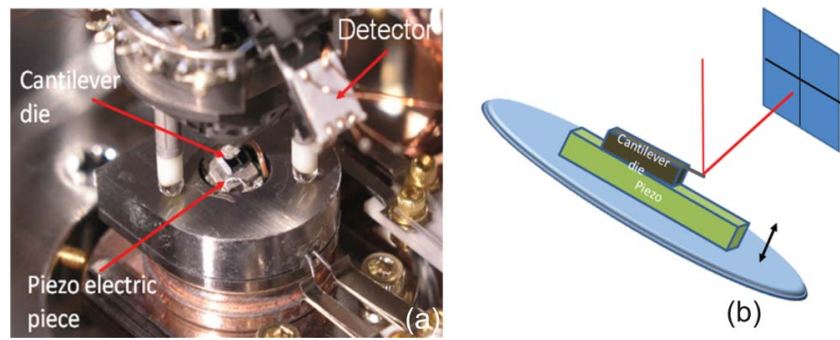

FIG. 3. (Color online) Custom-made AFM stage with UNCD cantilever die mounted on a tilted piezoceramic. This stage is inserted into a RHK AFM sample holder. A thermocouple is attached to the die to measure the temperature.

tilted at $22.5^{\circ}$ (so as to reflect incident laser light off of the cantilever to the photo detector) as shown in Fig. 3 (see schematic). Cantilever dies were mounted on top of the piezoceramic and the cantilever deflection was measured using the AFM photo detector. Resonant frequencies were identified by sweeping the input frequency to the piezo across the expected frequency range for the cantilever beams. To determine the quality factor, a ring-down measurement approach was employed whereby the excitation was stopped and the subsequent cantilever motion recorded using a Tektronix 3014B oscilloscope. An exponential fit to the ring-down curve gives a measure of dissipation in the fixed-free beams. The sample location was used instead of the regular cantilever location as it allowed us to independently cool the cantilever using the variable temperature sample stage of the AFM. Results at varying temperatures will be reported separately.

Dies without cantilevers were also studied to determine the biaxial modulus. $\mathrm{XeF}_{2}$ dry etching chemistry is used to produce an uninterrupted overhang of UNCD films grown on silicon. The amplitude and wavelength of the overhanging portion were measured using a Zygo white light scanning interferometer (NewView 6K). Different overhangs were produced until the film is completely relaxed to determine the biaxial modulus.

\section{RESULTS and DISCUSSION}

\section{A. Young's modulus}

The measured resonant frequencies of cantilevers of different lengths and overhangs are plotted in Fig. 4. The resonant frequency for the $n$th mode of an undamped freely vibrating cantilever is given by ${ }^{27}$

$$
f_{n}=\frac{\beta_{n}^{2}}{2 \pi} \frac{t}{L^{2}} \sqrt{\frac{E}{12 \rho}},
$$

where $L$ and $t$ are the length and thickness of the beam, respectively, and $E$ and $\rho$ are Young's modulus and density, respectively. $\beta_{n}$ is a constant equal to $\sim 1.875,4.694$, and 7.855 for $n=1,2,3$, respectively. Therefore, the Young's modulus of the cantilever material can be determined if the mass density and the dimensions are known. Measurements of resonance frequencies vs $1 / L^{2}$ for all cantilevers with overhangs ranging from 5 to $85 \mu \mathrm{m}$ are shown in Fig. 4(a).
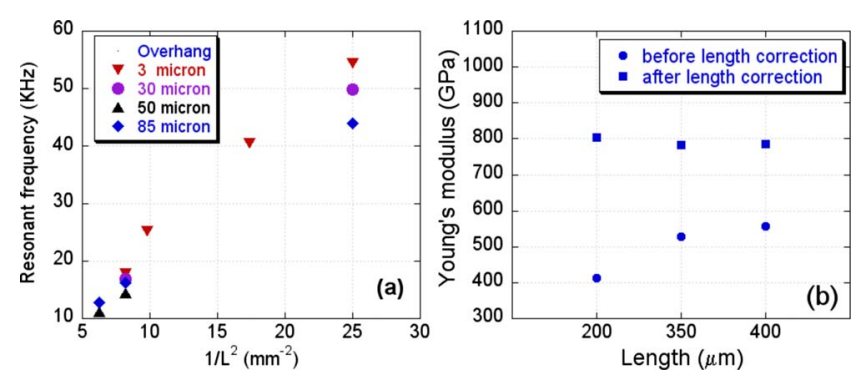

FIG. 4. (Color online) (a) First flexural resonance frequency of the cantilevers as a function of the inverse square of the cantilever length. (b) A comparison of the modulus determined from Eq. (9) as a function of actual cantilever length, before and after adding an effective length of $36 \mu \mathrm{m}$ to the actual length of the levers. All three cantilevers were on a single die with an $85 \mu \mathrm{m}$ overhang.

In principle, if the mass density is specified, Eq. (9) could then be used to determine Young's modulus. However, the presence of an overhang at the cantilever base alters the resonant frequency from the value predicted by Eq. (9) by an amount which increases as the lever gets shorter. Therefore, we employed two methods to correct for this effect. First, we determined the modulus numerically using the COMSOL multiphysics package (COMSOL Inc. Burlington, MA, USA), accounting for the measured overhang. This yielded a Young's modulus of $790 \pm 30 \mathrm{GPa}$. Second, the Young's modulus was independently estimated by adding an effective length chosen to give a constant Young's modulus for all the levers of different lengths. ${ }^{30}$ Figure 4(b) shows such an estimate of the Young's modulus for cantilevers with $85 \mu \mathrm{m}$ overhang obtained by adding $36 \mu \mathrm{m}$ to the actual length of the cantilevers. Based on this estimate, we determine the modulus to be $792 \pm 38 \mathrm{GPa}$.

Both of the calculations of the Young's modulus described above depend linearly on the density of the UNCD films as shown in Eq. (9). The density is not known with certainty. For the calculations presented here, the density of the UNCD is assumed to be equal to that of single-crystal diamond $\left(3500 \mathrm{~kg} / \mathrm{m}^{3}\right)$. The reported uncertainty of the Young's modulus of individual cantilevers was determined from the accuracy of the thickness of the beams measured in SEM, which leads to an error of $\sim 4 \%$ in the measured Young's modulus. The Hershey-Kroner-Eshelby averaging procedure for polycrystalline diamond predicts an isotropic Young's modulus of $1143 \mathrm{GPa} .{ }^{31}$ Thus, the measured Young's modulus is $\sim 25-30 \%$ lower than the theoretically calculated value for microcrystalline diamond with randomly oriented grains. ${ }^{31}$ Our measured value is $\sim 5-15 \%$ lower than the experimentally determined value for the modulus of UNCD films grown using MPCVD at $\sim 800{ }^{\circ} \mathrm{C}$. Measurements on these traditional UNCD were performed using nanoindentation $(864 \mathrm{GPa})^{5}$ and cantilever beam deflection (916-959 GPa). ${ }^{4}$ However, MPCVD-grown NCD fixed-fixed resonator structures yielded Young's modulus values ranging from 680-980 GPa, ${ }^{9-11}$ and MPCVD-grown UNCD fixed-fixed resonators grown at $550{ }^{\circ} \mathrm{C}$ yielded a modulus of $710 \mathrm{GPa}{ }^{2}$

The reasons for the somewhat lower Young's modulus of the UNCD films studied here compared to MPCVD grown traditional UNCD films discussed are not understood at this 
time. Theoretical studies ${ }^{32-34}$ of mechanical properties of UNCD have indicated that the observed Young's modulus strongly depends on the $s p^{2}$ content as well as volume fraction of grain boundaries. Philip et al. ${ }^{35}$ showed that NCD films (columnar grains, grain size 10-100 nm) can have Young's moduli in the range of 500-1120 GPa depending on the initial nucleation density. Thinner films of UNCD are reported to have a reduced Young's modulus compared to single-crystal diamond, ${ }^{36}$ possibly due to the enhanced influence of the inhomogeneous seed layer in the films which can be geometrically irregular and can possess enhanced $s p^{2}$ bonding. ${ }^{13,14}$ To understand the origins of these differences fully, it would be desirable to perform a systematic series of studies comparing UNCD films grown using HFCVD and MPCVD techniques at the same temperature, with similar chemistries, such that both films show similar content of $s p^{2}$ and $s p^{3}$ bonds, and comparable nanostructures, as well as to systematically vary the thickness. However, it can be speculated that the different Young's modulus observed for our films compared to traditional MPCVD UNCD films and MCD films may be attributed to the volume fraction of grain boundaries which accommodate some amount of disordered carbon, dangling bonds, and hydrogen, and possibly to the seeding method used and the resulting initial nucleation density.

\section{B. Poisson's ratio}

The biaxial modulus $\left(E_{b}\right)$ of the thin films can be estimated by measuring the period and the amplitude of the sinusoidal curvature of the fully relaxed overhang of the film if the residual compressive stress is known. ${ }^{37,38}$ To ensure the film is completely relaxed, the film has to be underetched until the ratio of amplitude to wavelength does not change with further underetching. For the fully relaxed thin films with sinusoidal amplitude $A_{0}$ much smaller than the wavelength, the biaxial modulus is given by 37,39

$$
\frac{E}{1-\nu} \approx\left(\frac{\lambda}{\pi A_{0}}\right)^{2} \sigma_{0},
$$

where $\sigma_{0}$ is the residual compressive stress and $\nu$ is the Poisson's ratio of the film. To eliminate the effects of boundaries, the overhanging ledge should be several wavelength long (in the direction parallel to the edge of the substrate), and the underetch should be more than half of the wavelength $(\lambda / 2)$ of relaxed overhang. If the Young's modulus and the residual compressive stress in the film are measured independently, then we can determine the Poisson's ratio $\nu$ using

$$
\nu=\frac{E_{b}-E}{E_{b}} .
$$

Residual stress in the film was relieved by undercutting the substrate using dry etching as described above. Figure 5 shows the ratio of the amplitude of the undulation $A_{0}$ to the period $\lambda$ of the overhanging portion of the film as a function of the overhang length. For overhangs less than $50 \mu \mathrm{m}$, periodic undulations are barely observed. Beyond an overhang of $160 \mu \mathrm{m}(\sim \lambda / 2)$, the ratio stabilizes to 0.0067 and thus

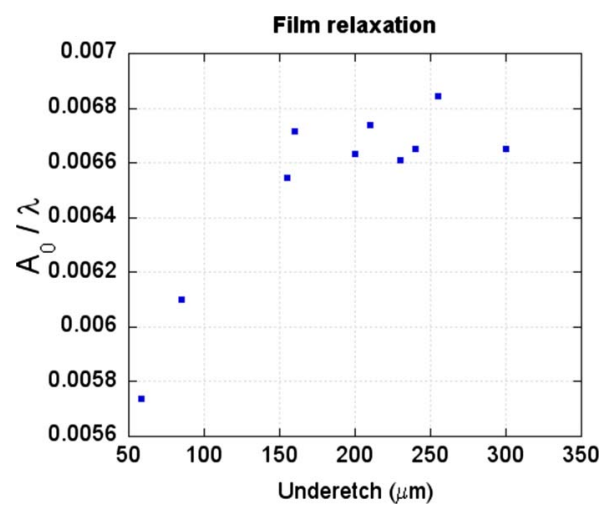

FIG. 5. (Color online) Ratio of amplitude to wavelength of the overhang as a function of the overhang length. For overhangs between 50 and $160 \mu \mathrm{m}$, the UNCD film is not fully relaxed from residual stress present in the substrate. Beyond $160 \mu \mathrm{m}, A_{0} / \lambda$ does not change with further etch depths.

the film is fully relaxed and the remaining substrate does not change the stress relaxation behavior of the edge of the film. Figure 6(a) shows the profile of one such overhang taken using scanning white light interferometer. Figure 6(b) shows the line profile of the overhang at the ridge of the sinusoidal undulation. Based on these measurements, we estimate the biaxial modulus to be $838 \pm 2 \mathrm{GPa}$. Using the Young's modulus of $790 \pm 30 \mathrm{GPa}$ determined using the resonance measurements described earlier, the calculated Poisson's ratio of UNCD is then $0.057 \pm 0.038$. The large relative error is due to the subtraction of two large, similar values $E$ and $E_{b}$ to produce a much smaller value.

The theoretical value of Poisson's ratio of polycrystalline diamond with randomly oriented grains and ignoring the contribution of grain boundaries is 0.069 , based on the Hershey-Kroner-Eshelby averaging method. ${ }^{31}$ A low Poisson's ratio is a unique property of tetrahedral $s p^{3}$ bonded carbon, which shows a higher elastic resistance to bond bending compared to bond stretching. However for UNCD films, which have a significant proportion of grain boundaries containing $s p^{2}$ bonded carbon, the Poisson's ratio may be expected to be higher than that for theoretically predicted value for polycrystalline diamond. Indeed, the measured Poisson's ratio of tetrahedral amorphous carbon (ta-C) films

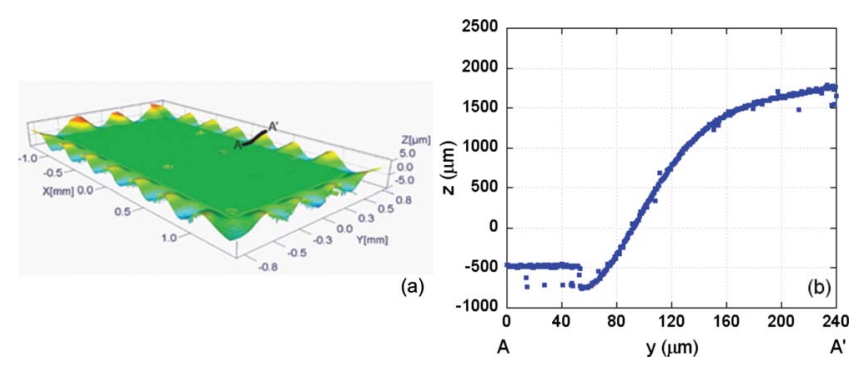

FIG. 6. (Color online) (a) Interferometric image of the UNCD film with a $200 \mu \mathrm{m}$ etched overhang. (b) Line scan of the section shown in 7 a showing a near saturation beyond $160 \mu \mathrm{m}$. The apparent jump in the height at the base of the overhang is simply due to the change in reflectivity from the more reflective underlying substrate. 

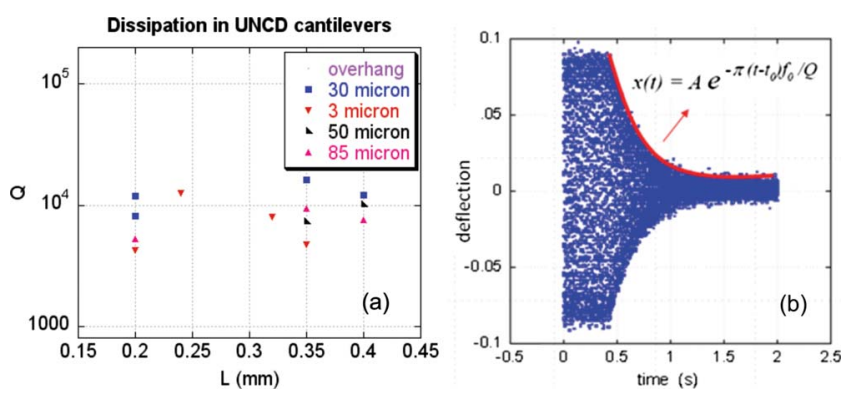

FIG. 7. (Color online) (a) Quality factor of UNCD cantilevers with different cantilever and overhang lengths. (b) Typical ringdown measurement with curve fitting equation.

containing much higher $s p^{2}$ content is $0.202 \pm 0.054 .{ }^{40} \mathrm{In}$ comparison with polycrystalline diamond, UNCD grain boundaries are atomically abrupt. ${ }^{2}$ However, because of the small grain size of UNCD, a significant fraction of atoms are in the grain boundaries or very close to the grain boundaries in UNCD. This significantly influences the bonding nature $^{41,42}$ and hence the mechanical behavior of the UNCD films. The influence on Poisson's ratio for this special case has just recently been modeled. A theoretical predictions for Poisson's ratio of UNCD (calculated from Young's modulus and the bulk modulus) yielded a value of 0.07 for a grain size of $4.41 \mathrm{~nm}$ with significant $(9.12 \%)$ non-diamond $\left(s p^{2}\right)$ content, ${ }^{34}$ substantially more than the $s p^{2}$ content of $4.3 \%$ that we measured for our films using NEXAFS. This suggests that a low Poisson's ratio can still be preserved even with significant $s p^{2}$ content. However, one limitation of this model is that it does not consider effects of the presence of hydrogen in grain boundaries. ${ }^{6}$ As well, note that the value of Poisson's ratio, like the value for $E$, does depend on the mass density, which could be lower for UNCD as compared to the value single-crystal diamond, which we have used in our calculations. A lower density reduces the calculated modulus and hence increases the measured Poisson's ratio.

\section{Dissipation in diamond}

Ring-down measurements were conducted at the fundamental flexural resonant frequencies of the cantilevers under UHV conditions. The curve fit to the damped oscillation yielded quality factors in the range of 5000-16000 for all the cantilevers of different dimensions and overhangs, with no specific dependence on the dimension of the cantilever and the overhang length, as shown in Fig. 7(a). Quality factors were observed to be stable with time. Figure 7(b) shows a typical ring-down measurement with a decaying exponential curve fit used for determining the dissipation in the resonators. We have measured quality factors up to 170000 on single-crystal silicon cantilevers of similar dimension on the same platform, which indicates that the dissipation due to the measuring technique is minimal. The absence of any metal coating on these cantilevers means that the observed dissipation can be attributed to clamping losses and/or intrinsic dissipation in the UNCD itself. With reference to Sec. II, clamping losses for our cantilever beams with overhangs are difficult to model. The low resonant frequencies $(\mathrm{kHz})$ of our

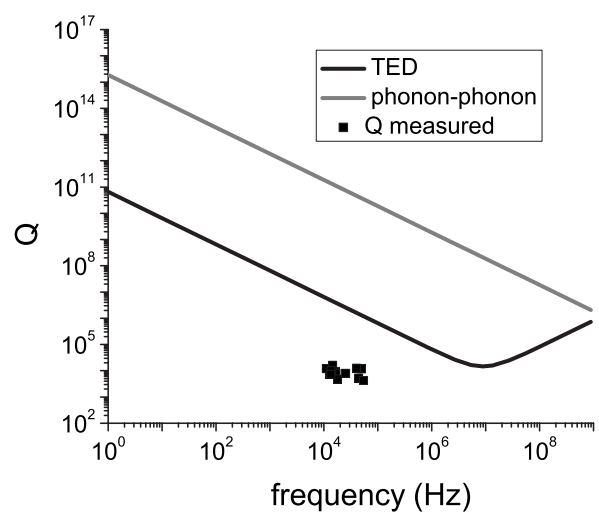

FIG. 8. Solid lines: calculated dissipation in UNCD cantilevers (thickness $=1 \mu \mathrm{m}$ ) due to TED, phonon-phonon dissipation as a function of frequency. Data points: measured dissipation at fundamental flexural resonance of the cantilevers used in this study. Since diamond remains an ideal Grüneisen's solid independent of the pressure, a Grüneisen's constant of 1 has been used in the models.

beams and high acoustic velocity of diamond result in the wavelength of the elastic wave in the base $\lambda_{b}$ being much higher than the thickness of the base $t_{b}$. We have thicknessmatching overhangs at the cantilever base $\left(t=t_{b}\right)$ and this thin overhanging membrane attaches to thick base (UNCD deposited on top of $\mathrm{Si}$ ). A more elaborate approach which considers the finite extent of our overhang and presence of different layers attached to this thin overhanging membrane are required to estimate the clamping losses. As mentioned earlier, we did not see a systematic dependence of quality factor on either the dimension of the beam or the length of the overhang. This strongly indicates that the losses in our beams are dominated by intrinsic dissipation mechanisms such as TED, internal friction due to defects, or dissipation due to interaction between thermal phonon and acoustic phonons.

The observed dissipation is at least 1000 times higher than the theoretical prediction for TED, phonon-phonon dissipation put together. The quality factor $Q$ vs frequency for UNCD cantilevers, jointly with $Q$ calculated for possible loss mechanisms, are all shown in Fig. 8. Table I shows the constants used for the estimation of dissipation in UNCD cantilevers that are plotted in Fig. 8. The comparison of $Q$ 's shown in Fig. 8 indicates that the dissipation is mainly due to the relaxation of large number of defects in the bulk or the surface of the film. Sepúlveda et al. ${ }^{24}$ reported a quality factor $Q$ for polycrystalline diamond (PCD) (grain size $\sim 300 \mathrm{~nm})$ fixed-free resonators in the range of 4000-100000, which includes values higher than the $Q$ 's observed for UNCD cantilevers with comparable dimensions reported in this paper. They suggested that, as the film percentage occupied by the nucleation layer containing fine grained diamond is increased, the quality factor of the resonators is reduced. ${ }^{43}$ This is consistent with the idea that the higher dissipation in the UNCD resonators described here is mainly due to the presence of the higher proportion of grain boundaries and defects. Hutchinson et al. ${ }^{10}$ measured the temperature dependence of dissipation of composite metal/ nanocrystalline diamond (NCD) fixed-fixed beams (colum- 
TABLE I. Constants used for estimating dissipation due to phonon-phonon dissipation, and thermoelastic dissipation.

\begin{tabular}{lc}
\hline \hline Parameter & Values used \\
\hline$C_{p}\left(\mathrm{~J} \mathrm{Kg}^{-1} \mathrm{~K}^{-1}\right)$ & $502^{\mathrm{a}}$ \\
$\alpha\left(\times 10^{6} \mathrm{~K}^{-1}\right)$ & $1^{\mathrm{a}}$ \\
$\gamma$ & $1^{\mathrm{a}}$ \\
$\mathrm{T}(\mathrm{K})$ & 300 \\
$C\left(\times 10^{6} \mathrm{~J} \mathrm{~m}^{-3} \mathrm{~K}^{-1}\right)$ & $1.767^{\mathrm{a}}$ \\
$\rho\left(\mathrm{Kg} / \mathrm{m}^{3}\right)$ & $3500^{\mathrm{a}}$ \\
$E(\mathrm{GPa})$ & 800 \\
$\kappa\left(\mathrm{W} \mathrm{m}{ }^{-1} \mathrm{~K}^{-1}\right)$ & 10 \\
& $11.357 ;$ \\
$v_{D} ; v\left(\times 10^{3} \mathrm{~m} \mathrm{~s}^{-1}\right)$ & 15.33 \\
\hline \hline
\end{tabular}

${ }^{a}$ Values used are for single-crystal diamond.

nar grains, grain size $5-15 \mathrm{~nm}$ ). These high-frequency $(\mathrm{MHz})$ resonators (dimensions $\sim 0.8 \mu \mathrm{m} \times 0.5 \mu \mathrm{m}$ $\times 10 \mu \mathrm{m}$ ) showed the presence of a Debye peak at $55 \mathrm{~K}$ and a dramatic increase in dissipation above $100 \mathrm{~K}$. However, they reported a rather lower quality factor $(\sim 3000$, at room temperature) than the low-frequency UNCD cantilever resonators studied here. Higher dissipation in the NCD resonators can be attributed to the fact that as the resonator dimensions become smaller, dissipation due to surface effects also play a role. ${ }^{15,16}$ This argument is supported by the investigations of scaling of dissipation with the dimension of the NCD fixed-fixed resonators, as reported by Imboden et al. ${ }^{44}$ Factors that contribute to the higher dissipation in highfrequency fixed-fixed flexural resonators may also include clamping losses, losses that occur at metal-diamond interfaces between metal-diamond interfaces, and the dissipation in the metal layer of the composite beams. ${ }^{15}$ Cantilever resonator structures made from tetrahedral amorphous carbon (ta-C), which have a significant $s p^{3}$ content, $\left(80 \% s p^{3}, 20 \%\right.$ $s p^{2}$ ) showed a lower $Q$ of $3500 .{ }^{45}$ Dissipation in ta-C indicated the processes dominated by two-level tunneling states (TLS). ${ }^{11}$ Recent investigation ${ }^{36}$ of the quality factor of metalcoated UNCD fixed-fixed beams at extremely low tempera- tures $(<10 \mathrm{~K})$ indicated the presence of TLS processes. Table II summarizes the results reported by other groups ${ }^{9-11,24,44,45}$ as compared to ours for different flexural beams fabricated using NCD, UNCD, or ta-C films.

The temperature dependence of the dissipation in our fixed-free beams will clarify the nature of dissipation in UNCD cantilever beams. This will be discussed in a separate publication. However, preliminary investigations point to the significant role of defects at grain boundaries and surfaces, indicating that the variation in $Q$ that we observe may be due to variations in the grain boundary or surface structure. This also suggests that controlling the grain boundaries and surfaces may be the key to controlling and increasing the $Q$ value.

\section{CONCLUSIONS}

The Young's modulus of medium-temperature $\left(680^{\circ} \mathrm{C}\right)$ HFCVD-grown UNCD films is measured to be $\sim 790 \pm 30 \mathrm{GPa}$. This value is approximately $25-30 \%$ lower than the theoretically predicted value for the Young's modulus of randomly oriented polycrystalline diamond having much larger grains and $\sim 15 \%$ lower than the experimentally determined value for the Young's modulus of traditional UNCD films. ${ }^{4}$ However, it is significantly higher than values measured for metal-coated UNCD beams grown at lower temperature. $^{2}$ Non-diamond imperfections in the carbon atom bonding (disordered carbon, dangling bonds, hydrogen, and increased $s p^{2}$ bonding) at the grain boundaries is the likely cause of the reduction in the modulus in UNCD films. The biaxial Young's modulus of the films was determined to be $838 \pm 2$ GPa by measuring the amplitude and wavelength of fully relaxed substrate-free overhangs of the compressively stressed films. Based on these measurements, the Poisson's ratio was estimated for the first time for any UNCD films to be $0.057 \pm 0.038$. Dissipation in the UNCD cantilevers is determined using ring-down measurements under UHV conditions and ranged from 5000-16000 at $\sim \mathrm{kHz}$ resonance frequencies. The UNCD cantilever resonators exhibited higher dissipation compared to PCD cantilevers with comparable resonant frequencies, but less dissipation than

TABLE II. Room temperature quality factor of flexural resonators from our work, as well as reported by other groups.

\begin{tabular}{lccccc}
\hline \hline Structure & $\begin{array}{c}\text { Resonant } \\
\text { frequency }\end{array}$ & Material & $Q$ & $\begin{array}{c}\text { Growth } \\
\text { technique }\end{array}$ & $\begin{array}{c}\text { Actuation } \\
\text { method }\end{array}$ \\
\hline Fixed-fixed $^{\mathrm{a}}$ & $17-66 \mathrm{MHz}$ & $\mathrm{NCD}$ & $600-2400$ & MPCVD & Magnetomotive \\
Fixed-free $^{\mathrm{b}}$ & $8-50 \mathrm{KHz}$ & $\mathrm{PCD}$ & $4000-100000$ & MPCVD & $\begin{array}{c}\text { Piezoelectric } \\
\text { Doubly clamped paddle }^{\mathrm{c}}\end{array}$ \\
Fixed-free (our work) $^{6-30 \mathrm{MHz}}$ & $\mathrm{NCD}$ & $2400-3500$ & MPCVD & Piezoelectric \\
Fixed-fixed $^{\mathrm{d}}$ & $12-45 \mathrm{KHz}$ & $\mathrm{UNCD}$ & $5000-16000$ & HFCVD & Piezoelectric \\
Fixed-free $^{\mathrm{e}}$ & $14-157 \mathrm{MHz}$ & $\mathrm{NCD}$ & $\sim 3000$ & MPCVD & Magnetomotive \\
\hline \hline
\end{tabular}

${ }^{\text {aReference } 44 .}$

${ }^{b}$ Reference 24.

cReference 9.

${ }^{\mathrm{d}}$ Reference 10.

${ }^{\text {e}}$ Reference 45. 
amorphous carbon cantilever resonators. ${ }^{45}$ We attribute dissipation in the UNCD resonators mainly to the presence of defects such as nondiamond bonding at grain boundaries and surfaces.

\section{ACKNOWLEDGMENTS}

This project was supported by DARPA under Grant No.
06-W238 and the U.S. Department of Energy under Contract No. DE-AC02-06CH05117. The work at ANL was supported by the U. S. Department of Energy, Office of Science, Office of Basic Energy Sicences under Contract No. DE-AC0206CH11357. We thank A. R. Konicek for assistance with analyzing the NEXAFS spectra. We thank K. Ekinci for useful discusions of this work.
${ }^{1}$ N. N. Naguib, J. W. Elam, J. Birrell, J. Wang, D. S. Grierson, B. Kabius, J. M. Hiller, A. V. Sumant, R. W. Carpick, O. Auciello, and J. A. Carlisle, Chem. Phys. Lett. 430, 345 (2006).

${ }^{2}$ O. Auciello, S. Pacheco, A. V. Sumant, C. Gudeman, S. Sampath, A. Datta, R. W. Carpick, V. P. Adiga, P. Zurcher, M. Zhenqiang, Y. Hao-Chih, J. A. Carlisle, B. Kabius, J. Hiller, and S. Srinivasan, IEEE Microw. Mag. 8, 61 (2007).

${ }^{3}$ A. R. Krauss, O. Auciello, D. M. Gruen, A. Jayatissa, A. Sumant, J. Tucek, D. C. Mancini, N. Moldovan, A. Erdemir, D. Ersoy, M. N. Gardos, H. G. Busmann, E. M. Meyer, and M. Q. Ding, Diamond Relat. Mater. 10, 1952 (2001).

${ }^{4}$ H. D. Espinosa, B. C. Prorok, B. Peng, K. H. Kim, N. Moldovan, O. Auciello, J. A. Carlisle, D. M. Gruen, and D. C. Mancini, Exp. Mech. 43, 256 (2003).

${ }^{5}$ A. V. Sumant et al., in Materials Science of Microelectromechanical Systems (MEMS) Devices III, edited by M. deBoer, M. Judy, H. Kahn, and S. M. Spearing, MRS Proceedings, Vol. 657, Symposium EE, (Materials Research Society, Pittsburgh, 2000) p. EE5.33.1.

${ }^{6}$ C. Liu, X. C. Xiao, J. Wang, B. Shi, V. P. Adiga, R. W. Carpick, J. A. Carlisle, and O. Auciello, J. Appl. Phys. 102, 074115 (2007).

${ }^{7}$ A. Gaidarzhy, M. Imboden, P. Mohanty, J. Rankin, and B. W. Sheldon, Appl. Phys. Lett. 91, 203503 (2007).

${ }^{8}$ C. F. Wang, Y. S. Choi, J. C. Lee, E. L. Hu, J. Yang, and J. E. Butler, Appl. Phys. Lett. 90, 081110 (2007).

${ }^{9}$ L. Sekaric, J. M. Parpia, H. G. Craighead, T. Feygelson, B. H. Houston, and J. E. Butler, Appl. Phys. Lett. 81, 4455 (2002).

${ }^{10}$ A. B. Hutchinson, P. A. Truitt, K. C. Schwab, L. Sekaric, J. M. Parpia, H. G. Craighead, and J. E. Butler, Appl. Phys. Lett. 84, 972 (2004).

${ }^{11}$ T. H. Metcalf, X. Liu, B. H. Houston, J. W. Baldwin, J. E. Butler, and T. Feygelson, Appl. Phys. Lett. 86, 081910 (2005).

${ }^{12}$ A. R. Konicek, D. S. Grierson, P. U. P. A. Gilbert, W. G. Sawyer, A. V. Sumant, and R. W. Carpick, Phys. Rev. Lett. 100, 235502 (2008).

${ }^{13}$ A. V. Sumant, D. S. Grierson, J. E. Gerbi, J. A. Carlisle, O. Auciello, and R. W. Carpick, Phys. Rev. B 76, 235429 (2007).

${ }^{14}$ A. V. Sumant, D. S. Grierson, J. E. Gerbi, J. Birrell, U. D. Lanke, O. Auciello, J. A. Carlisle, and R. W. Carpick, Adv. Mater. (Weinheim, Ger.) 17, 1039 (2005).

${ }^{15}$ C. Seoanez, F. Guinea, and A. H. Castro Neto, Phys. Rev. B 77, 125107 (2008).

${ }^{16}$ P. Mohanty, D. A. Harrington, K. L. Ekinci, Y. T. Yang, M. J. Murphy, and M. L. Roukes, Phys. Rev. B 66, 085416 (2002).

${ }^{17}$ S. Bianco, M. Cocuzza, S. Ferrero, E. Giuri, G. Piacenza, C. F. Pirri, A. Ricci, L. Scaltrito, D. Bich, A. Merialdo, P. Schina, and R. Correale, J. Vac. Sci. Technol. B 24, 1803 (2006).
${ }^{18}$ M. C. Cross and R. Lifshitz, Phys. Rev. B 64, 085324 (2001).

${ }^{19}$ D. M. Photiadis and J. A. Judge, Appl. Phys. Lett. 85, 482 (2004).

${ }^{20}$ Y. Jimbo and K. Itao, J. Horol. Inst. Jpn. 47, 1 (1968).

${ }^{21}$ C. Zener, Phys. Rev. 53, 90 (1938).

${ }^{22}$ R. Lifshitz and M. L. Roukes, Phys. Rev. B 61, 5600 (2000).

${ }^{23}$ V. T. Srikar and S. D. Senturia, J. Microelectromech. Syst. 11, 499 (2002).

${ }^{24}$ N. Sepulveda, L. Jing, D. M. Aslam, and J. P. Sullivan, J. Microelectromech. Syst. 17, 473 (2008).

${ }^{25}$ V. B. Braginsky, V. P. Mitrofanov, and V. I. Panov, Systems with small Dissipation (University of Chicago Press, Chicago, 1985).

${ }^{26}$ M. A. Angadi, T. Watanabe, A. Bodapati, X. C. Xiao, O. Auciello, J. A. Carlisle, J. A. Eastman, P. Keblinski, P. K. Schelling, and S. R. Phillpot, J. Appl. Phys. 99, 114301 (2006).

${ }^{27}$ A. N. Cleland, Foundations of Nanomechanics (Springer, Heidelberg, New York, 2003).

${ }^{28}$ C. Lenardi, P. Piseri, V. Briois, C. E. Bottani, A. L. Bassi, and P. Milani, J. Appl. Phys. 85, 7159 (1999).

${ }^{29}$ R. J. Cannara, M. J. Brukman, K. Cimatu, A. V. Sumant, S. Baldelli, and R. W. Carpick, Science 318, 780 (2007).

${ }^{30}$ E. Q. J. Mencik, J. Mater. Res. 14, 2152 (1999).

${ }^{31}$ C. A. Klein, Mater. Res. Bull. 27, 1407 (1992).

${ }^{32}$ J. T. Paci, T. Belytschko, and G. C. Schatz, Chem. Phys. Lett. 414, 351 (2005).

${ }^{33}$ J. T. Paci, T. Belytschko, and G. C. Schatz, Phys. Rev. B 74, 184112 (2006)

${ }^{34}$ I. N. Remediakis, G. Kopidakis, and P. C. Kelires, Acta Mater. 56, 5340 (2008).

${ }^{35}$ J. Philip, P. Hess, T. Feygelson, J. E. Butler, S. Chattopadhyay, K. H. Chen, and L. C. Chen, J. Appl. Phys. 93, 2164 (2003).

${ }^{36}$ M. Imboden and P. Mohanty, Phys. Rev. B 79, 125424 (2009).

${ }^{37}$ R. T. Howe and R. S. Muller, J. Appl. Phys. 54, 4674 (1983).

${ }^{38}$ P. G. Borden, Appl. Phys. Lett. 36, 829 (1980).

${ }^{39}$ S.-J. Cho, J.-W. Chung, and K.-R. Lee, Diamond Relat. Mater. 14, 1270 (2005).

${ }^{40} \mathrm{~S}$. Cho, I. Chasiotis, T. A. Friedmann, and J. P. Sullivan, J. Micromech. Microeng. 15, 728 (2005).

${ }^{41}$ C. Q. Sun, Prog. Solid State Chem. 35, 1 (2007).

${ }^{42}$ M. X. Gu, C. Q. Sun, Z. Chen, T. C. Au Yeung, S. Li, C. M. Tan, and V. Nosik, Phys. Rev. B 75, 125403 (2007).

${ }^{43}$ N. Sepulveda, D. M. Aslam, and J. P. Sullivan, Nineteenth IEEE International Conference on Micro Electro Mechanical Systems, MEMS 2006 Istanbul, 2006, p. 238.

${ }^{44}$ M. Imboden, P. Mohanty, A. Gaidarzhy, J. Rankin, and B. W. Sheldon, Appl. Phys. Lett. 90, 173502 (2007).

${ }^{45}$ D. A. Czaplewski, J. P. Sullivan, T. A. Friedmann, and J. R. Wendt, Diamond Relat. Mater. 15, 309 (2006). 